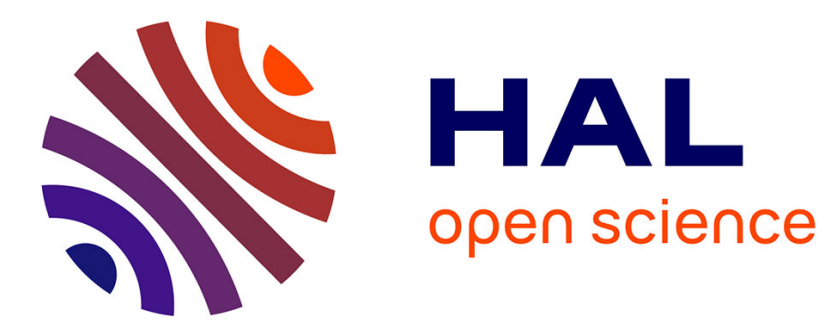

\title{
Raconter saint Antoine à Lisbonne. Acteurs, performances et effets du discours hagiographique contemporain \\ Cyril Isnart
}

\section{- To cite this version:}

Cyril Isnart. Raconter saint Antoine à Lisbonne. Acteurs, performances et effets du discours hagiographique contemporain. Cahiers de Littérature Orale, 2016, 79, https://clo.revues.org/2656. 10.4000/clo.2656 . halshs-01425142

\section{HAL Id: halshs-01425142 \\ https://shs.hal.science/halshs-01425142}

Submitted on 3 Jan 2017

HAL is a multi-disciplinary open access archive for the deposit and dissemination of scientific research documents, whether they are published or not. The documents may come from teaching and research institutions in France or abroad, or from public or private research centers.
L'archive ouverte pluridisciplinaire HAL, est destinée au dépôt et à la diffusion de documents scientifiques de niveau recherche, publiés ou non, émanant des établissements d'enseignement et de recherche français ou étrangers, des laboratoires publics ou privés. 


\section{Raconter saint Antoine à Lisbonne. Acteurs, performances et effets du discours hagiographique contemporain}

Telling the Life of Saint Anthony in Lisbon. Actors, Performances and Effects of Contemporary Hagiographic Discourse

Contando Santo António em Lisboa. Atores, performances e efeitos do discurso hagiográfico contemporâneo

\section{Cyril Isnart}

\section{grevues.org}

Édition électronique

URL : http://clo.revues.org/2656

ISBN : 9782858312344

ISSN : 2266-1816

\section{Éditeur}

INALCO

\section{Édition imprimée}

Date de publication : 1 janvier 2016

ISBN : 9782858312337

ISSN : 0396-891X

Référence électronique

Cyril Isnart, «Raconter saint Antoine à Lisbonne. Acteurs, performances et effets du discours hagiographique contemporain », Cahiers de littérature orale [En ligne], 79 | 2016, mis en ligne le 19 décembre 2016, consulté le 23 décembre 2016. URL : http://clo.revues.org/2656 ; DOI : 10.4000/ clo.2656

Ce document est un fac-similé de l'édition imprimée.

\section{cc) ()ㅇㅇ}

Cahiers de littérature orale est mis à disposition selon les termes de la licence Creative Commons Attribution - Pas d'Utilisation Commerciale - Partage dans les Mêmes Conditions 4.0 International. 


\title{
Raconter saint Antoine à Lisbonne. Acteurs, performances et effets du discours hagiographique contemporain
}

\author{
Cyril IsNART \\ Institut d'Ethnologie méditerranéenne, européenne et comparative, \\ UMR 7307, CNRS Aix Marseille Université
}

Saint Antoine de Padoue (c. 1191-1231), l'un des saints catholiques dont le culte est le plus largement diffusé dans le monde et qui a son plus important sanctuaire en Italie, est né à Lisbonne, au Portugal. C'est dans ce pays qu'il suit une formation intellectuelle et religieuse exceptionnelle et qu'il se dédie à la vie monastique jusqu'à l'aube de ses trente ans. Il rencontre alors le mouvement franciscain et décide de quitter son pays pour prêcher selon les principes de saint François. Dix ans plus tard, Antoine décède en Italie, où il est devenu l'un des piliers de l'ordre du saint d'Assise, théologien de l'Église catholique, prédicateur enthousiaste et thaumaturge reconnu. Il est canonisé en 1232, un an après sa mort, ses miracles et son œuvre concourant à la propagation de son culte à partir de Padoue. Au Portugal, il est le patron secondaire du pays, Lisbonne le considère comme le patron de la ville et il se trouve au centre des fêtes municipales du mois de juin (Pereira, 2015). Les pouvoirs politiques, depuis la fin du Moyen Âge, lui ont donné une place de choix dans le roman national et parmi les grands hommes portugais. Son image est incontournable aux côtés de celle de Notre-Dame de Fátima dans les églises, sur les autels domestiques et sur les étals des magasins des zones touristiques.

Le récit de sa vie tel que le transmettent les Bollandistes (1688, p. 705-716), savants bénédictins qui compilent les récits hagiographiques depuis le XVII ${ }^{\mathrm{e}}$ siècle, s'articule autour de trois périodes (la formation intellectuelle et religieuse au Portugal, la vocation franciscaine, la mission en Italie et en France) qui laissent 
relativement peu de place au Portugal et déroulent longuement les dix dernières années relatant ses miracles et ses activités de dirigeant ecclésiastique. Dans son pays natal, il est l'objet d'une attention particulière de la part des historiens et des savants religieux. L'article le concernant dans l'encyclopédie lusophone de référence du milieu du $\mathrm{XX}^{\mathrm{e}}$ siècle compte plus de soixante références bibliographiques, au-delà des éditions des écrits du saint (GEPB, 1936-1960, II, p. 836-856). Dans les versions imprimées que l'on trouve dans les publications destinées au grand public, sur les sites internet ou dans les guides touristiques, on note de plus amples développements sur la première période, avec des détails sur les lieux et les communautés religieuses fréquentés par le jeune religieux.

Cet article ${ }^{1}$ tente de décrire et d'analyser comment s'opère aujourd'hui, sur le lieu de son culte principal au Portugal, la qualification d'un saint Antoine de Padoue universel en un personnage surnaturel reconnu comme portugais. La réflexion s'appuiera sur une ethnographie du lieu de naissance supposé du saint à Lisbonne depuis 2012. Ce sanctuaire voit graviter autour de lui un ensemble d'acteurs religieux, touristiques et patrimoniaux dont le dénominateur commun est la figure de saint Antoine dans sa version portugaise. Quels sont les porteurs de ces récits hagiographiques ? À travers quels mécanismes narratifs le saint de Padoue demeure-t-il, à Lisbonne, le saint portugais par excellence ? Quels sont les effets sociaux et culturels de ces narrations contemporaines de la vie de saint Antoine?

Ce cas permet de reformuler plusieurs des problématiques classiques de l'anthropologie religieuse, notamment celles des premiers sociologues des religions tels que Robert Hertz (2014 [1913], p. 181-236), Stefan Czarnowski (1919), Henri Hubert (1919) et Maurice Halbwachs (1950). À travers une ethnographie contemporaine, il s'agit d'appréhender par exemple comment le récit contem-

1. Ce texte est le résultat des enquêtes soutenues par la Fundação para a Ciência e a Tecnologia (Portugal) dans le cadre du programme MERAP-MED « Religious Memories and Heritage Practices in the Mediterranean. Confessional Coexistence and Heritage Assertion » PTDC/IVC-ANT/4033/2012. Il constitue une version développée de deux communications présentées à l'atelier Eurethno « Le tourisme religieux en Europe », Perugia, septembre 2014 et à la journée d'étude du programme MERAP-MED « Lieux de culte : transferts, refondations et patrimonialisations en Méditerranée », Aixen-Provence, novembre 2014. Je remercie les participants à ces rencontres et les membres du programme de recherche, qui ont bien voulu commenter certains points présentés ici. Ma reconnaissance va à Pedro Teotónio Pereira et frei Pedro Nuno Antunes Fereira pour leur accueil et leurs conversations au musée et au sanctuaire. Que soient remerciés Norig Neveu, Josiane Bru, les éditrices de ce numéro, les lecteurs anonymes, ainsi que le directeur du Musée de Saint-Antoine, qui ont su proposer des améliorations aux premières versions de ce texte. 
porain de la vie d'un saint permet de comprendre la construction d'une figure collective emblématique, de liens entre politique et religion, ou d'un territoire partagé. Pour autant, l'analyse des usages rituels, culturels et politiques du saint de Lisbonne contribue à des interrogations sur les croisements entre tourisme et religion, notamment dans le cadre de l'anthropologie du pèlerinage et du tourisme (Badone et Roseman, 2004), et entre la religion et le patrimoine culturel (Paine, 2013 ; Faltrauer, Martin et Obadia, 2012 ; Isnart, 2009 ; 2014). Ainsi, au-delà de l'ethnographie d'une hagiographie dans un contexte contemporain, cette contribution vise à mieux saisir ce que la narration de la vie du saint, portée par des acteurs sensiblement différents, peut nous apprendre non seulement sur les façons de « faire » le saint, mais encore sur les superpositions contemporaines entre tourisme, patrimoine et religion.

\section{La vie portugaise de saint Antoine}

Saint Antoine ${ }^{2}$, ou Fernando de Bulhões de son nom de baptême, est né dans le quartier de la cathédrale de Lisbonne, à la fin du XII ${ }^{\mathrm{e}}$ siècle, dans une famille aisée et dans une ville depuis peu « reprise aux Maures ${ }^{3}$ par les rois catholiques. Il étudie à l'école de la cathédrale, puis se consacre à l'étude religieuse en entrant dans l'ordre des Augustins de la ville. Il s'installe ensuite à Coimbra, centre intellectuel et religieux du centre du pays, où il approfondit ses connaissances théologiques et philosophiques, tout en cultivant un art oratoire qui le distinguera de ses compagnons. C'est dans cette ville, en 1220, qu'il apprend que des Franciscains évangélisant le Maroc y ont trouvé la mort, devenant ce que la famille franciscaine nomme les « martyrs du Maroc ${ }^{4}$. Il décide de rejoindre alors l'ordre des frères mineurs et de partir à son tour au Maroc pour prêcher l'Évangile. Il y tombe malade rapidement et se voit contraint de revenir au Portugal. Son bateau est pourtant détourné par une tempête et il échoue en Sicile, d'où il part à la rencontre de saint François. Le fondateur de l'ordre lui donnera plusieurs missions d'évangélisation en France

2. Ce résumé constitue ma propre version de la vie du saint, rédigée pour cet article en synthétisant plusieurs récits que j'ai pu entendre pendant les enquêtes de terrain et plusieurs textes (brochures de l'Église, notices hagiographiques, dépliants touristiques, cartels du musée, etc.) auxquels j'ai pu avoir accès.

3. Au Portugal, comme en Espagne, la mythologie nationale a conservé le terme de « reconquête » (Reconquista) pour évoquer la prise de pouvoir des rois chrétiens sur les Arabo-musulmans au cœur du Moyen Âge.

4. Fêtés le 16 janvier (Bollandistes, 1643, p. 62-65), voir PAcheco, 2009 et Pestre de Almeida, 2009. 
et en Italie, notamment pour combattre des mouvements hérétiques dans le sud de la France. François reconnaîtra, malgré sa méfiance à l'égard du savoir et des livres (Le Goff, 1999), le talent d'orateur savant d'Antoine et la place nécessaire de l'étude dans le franciscanisme. Antoine meurt en 1231, près de Padoue, il est canonisé un an après, sa tombe devenant le lieu de nombreux miracles. Il reçoit en 1946 le titre de docteur de l'Église, qui distingue les penseurs de la doctrine catholique.

Le succès universel de saint Antoine est sans doute dû aux efforts des Franciscains pour inciter au culte de l'un des plus célèbres d'entre eux, comme à la volonté du pape Léon XIII, à la fin du XIX ${ }^{\mathrm{e}}$ siècle, d'assurer une meilleure place au saint de Padoue, mais également à la capacité de saint Antoine à s'intégrer dans des contextes catholiques (Roger, 1971 ; Van Dick, 1980, Brévot Dromzée, 2005 ; Menezes, 2004) ou non catholiques (notamment en Méditerranée, Albera et Fliche, 2012, Isnart, 2014), en Afrique (Thornton, 1998) ou en Inde (Sebastia, 2004).

Dans les territoires lusophones, les clercs, les spécialistes d'histoire religieuse et les fidèles le nomment saint Antoine de Lisbonne, aussi bien dans la littérature scientifique que dans les usages coutumiers. Le pouvoir politique portugais, depuis la monarchie jusqu'au régime autoritaire de Salazar, lui a toujours donné une place centrale, en privilégiant son sanctuaire, ou en l'enrôlant comme soldat dans les combats militaires que le pays menait. On raconte que les cloches de Lisbonne auraient sonné lors de sa canonisation en Italie. Le sénat médiéval de la ville se réunissait dans le sanctuaire, qui est devenu église royale pendant la période moderne. Dans l' « Exposition du Monde Portugais », que le pouvoir autoritaire de l'Estado Novo avait organisée en 1940 pour valoriser la présence coloniale et les richesses artisanales et artistiques du pays, saint Antoine faisait logiquement partie des grands hommes portugais mis à l'honneur, aux côtés des explorateurs du Nouveau Monde. Une reconstitution de sa maison natale, une messe de bénédiction et une exposition de sculptures permettaient de l'intégrer dans le panthéon politico-religieux du régime (Sequeira et Brasão (dir.), 1940). L'attachement à l'origine portugaise du saint se manifeste par ailleurs dans le soin que la province franciscaine du pays et la municipalité de Lisbonne procurent au lieu supposé de sa naissance, rapidement transformé en lieu de pèlerinage. Bien que le lieu de culte original ait été détruit par un tremblement de terre en 1755 , le sanctuaire reconstruit à la fin du XVIII ${ }^{\mathrm{e}}$ siècle (Silva, 1991) contient aujourd'hui une crypte que l'on considère comme la pièce où il serait né (figure 1). Situé dans le centre-ville, l'ensemble architectural que l'on nomme igreja-casa de Santo António (ou église-maison de saint Antoine), compte la chambre natale, une petite basilique, les pièces de la fraternité franciscaine qui anime le sanctuaire et un musée municipal. Il s'agit 
d'un haut lieu religieux et touristique de la ville de Lisbonne, que traversent des milliers de pèlerins étrangers, de fidèles lisboètes ou de touristes. Lors des fêtes municipales, au mois de juin, le sanctuaire devient le centre religieux de Lisbonne, et l'on compte jusqu'à 10000 visiteurs par jour pendant la treizaine de la fête de saint Antoine, entre le $1^{\text {er }}$ et le 13 juin. S'y déplacent également au cours de l'année, sur le mode du pèlerinage votif, des jeunes gens et des jeunes filles demandant l'aide du saint pour leur mariage, souvent en glissant un papier sous le tableau le représentant. Depuis les années 1950, la municipalité de Lisbonne offre à des couples défavorisés de la ville leur cérémonie de mariage, retransmise aujourd'hui à la télévision. Cette séquence fait partie intégrante de la fête municipale annuelle, aux côtés des bals et de la consommation de bière et de sardines grillées. Le rôle de saint marieur, on y reviendra, est une caractéristique valorisée de saint Antoine dans la péninsule ibérique et au Brésil (Menezes, 2004, p. 161).

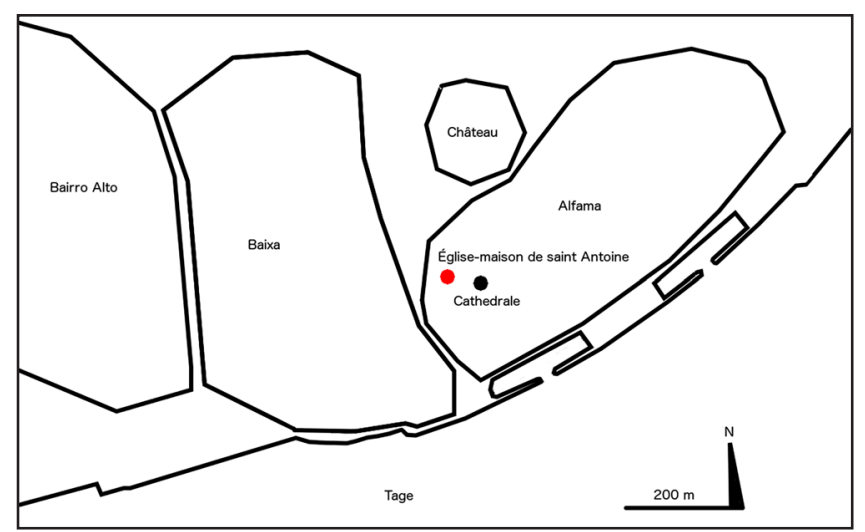

Figure 1 : Localisation de l'église-maison de saint Antoine à Lisbonne (c) Cartographie C. Isnart et Google Maps

\section{Qui parle de saint Antoine?}

Comme c'est le cas dans de nombreux lieux de pèlerinage, personne ne possède le monopole de la narration de la vie de saint Antoine à Lisbonne. Eade et Sallnow (1991, p. 15-16) ont souligné que les sanctuaires ne sont pas le support d'une fonction unique et d'un sens universellement partagé. Au contraire, les pèlerins, dans leur diversité, viennent donner au lieu une signification qui est largement déterminée par leurs propres expériences et leurs propres attentes. On peut en dire autant des acteurs responsables des sanctuaires catholiques au contact des pèlerins et des touristes de passage. L'ethnographie à Lisbonne montre que coexistent plu- 
100 Des vies extraordinaires : les territoires du récit $-\mathrm{n}^{\circ} 79$

sieurs types de narrateurs qui ne s'opposent pas frontalement, mais qui profèrent, chacun pour leur public, une interprétation, à but religieux, patrimonial ou touristique, plus ou moins conforme à la « vulgate » de la vie du saint.

Le premier ensemble de narrateurs est constitué par les responsables franciscains du sanctuaire (figure 2). Si le bâtiment semble avoir appartenu à la municipalité de Lisbonne depuis le Moyen Âge, sa gestion a été confiée à la province franciscaine du Portugal le 14 septembre 1926. Après la période libérale et anticléricale de la Première République, qui aboutit à l'extinction des ordres religieux et à la nationalisation de leurs biens, l'instauration d'un pouvoir autoritaire plus favorable à l'Église catholique a sensiblement contribué à la relance du culte du saint et à la restauration de son sanctuaire. La fonction d'entretien et d'animation du lieu de culte est aujourd'hui assurée par une petite fraternité de frères franciscains mineurs (OFM, qui résident au sanctuaire), une dizaine de membres de l'ordre franciscain séculier (OFS) qui occupent un poste de travail au sanctuaire, et quelques laïcs. Les frères mineurs célèbrent la messe deux fois par jour. Ces cérémonies constituent, en plus de leurs fonctions rituelles, des moments de narration de la vie du saint, de l'histoire du lieu et de la mémoire de la communauté locale. L'un des frères séculiers est chargé plus spécialement de l'organisation des services religieux et de l'accueil des visiteurs et des pèlerins. C'est vers lui que sont orientés les guides et leurs groupes qui visitent le sanctuaire, ainsi que les prêtres qui souhaitent célébrer une messe avec leur groupe de pèlerins. Il est également missionné pour le contact avec les médias, la municipalité, le musée adjacent et les associations de guides locaux. L'orientation générale du sanctuaire s'inscrit dans ce que l'on appelle le « tourisme religieux » (ou la « pastorale du tourisme » dans le langage catholique), qui entretient des liens forts avec le pèlerinage (SaintMartin, 2013). 


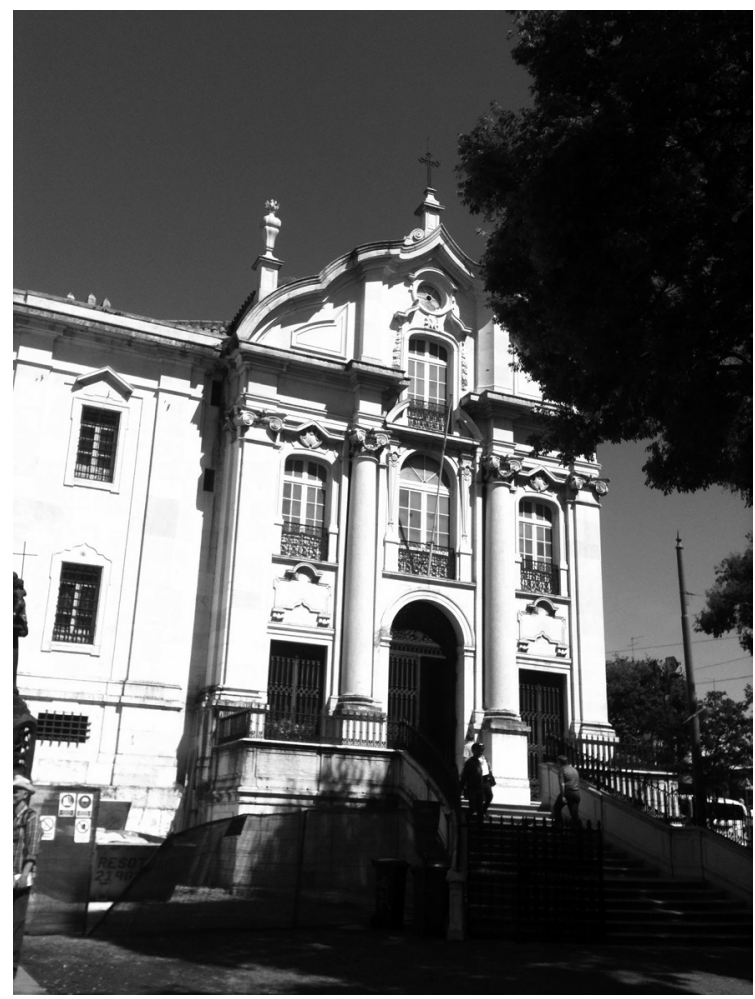

Figure 2

Église-maison de saint Antoine, Lisbonne, 2013

(C) Photographie de l'auteur

Le second foyer de narration de la vie du saint se situe dans le musée municipal attenant à l'église, consacré à la figure d'Antoine. Le musée (comme le sanctuaire) est propriété de la municipalité de Lisbonne, qui a mené à bien sa conception et son ouverture en 1962. Le projet de consacrer un espace d'exposition au saint de Lisbonne remonte à la première moitié $\mathrm{du} \mathrm{Xx}^{\mathrm{e}}$ siècle, alors que la ville devient une destination touristique internationale sous l'impulsion du pouvoir autoritaire de l'Estado Novo. Dans les projets antérieurs, c'est parfois l'église elle-même qui devait devenir le lieu d'exposition (par exemple Pinto, 1942). Mais c'est dans les années 1960, après la donation de collections privées d'images de saint Antoine et avec la volonté municipale de le valoriser comme emblème de la ville, que le Museu Antoniano ouvre ses portes dans un bâtiment adjacent au sanctuaire. La 
dernière restructuration, menée au début des années 2010, a permis de repenser et d'actualiser l'image un peu désuète du musée des années 1960. Le parcours muséographique, les objets exposés et les activités de guidage par des membres du service culturel (figure 3) de la ville de Lisbonne, renouvelés depuis la réouverture en juillet 2014, tentent de transmettre la vie du saint et sa place dans la culture du Portugal selon des paramètres qui relèvent à la fois du tourisme et de la valorisation des ressources culturelles municipales. Le directeur du musée a rédigé une notice explicative que suivent les guides de la municipalité et qui contient la chronologie, le contexte historique, la diffusion du culte, les traditions populaires et un ensemble de dictons, citations et anecdotes concernant saint Antoine au Portugal.

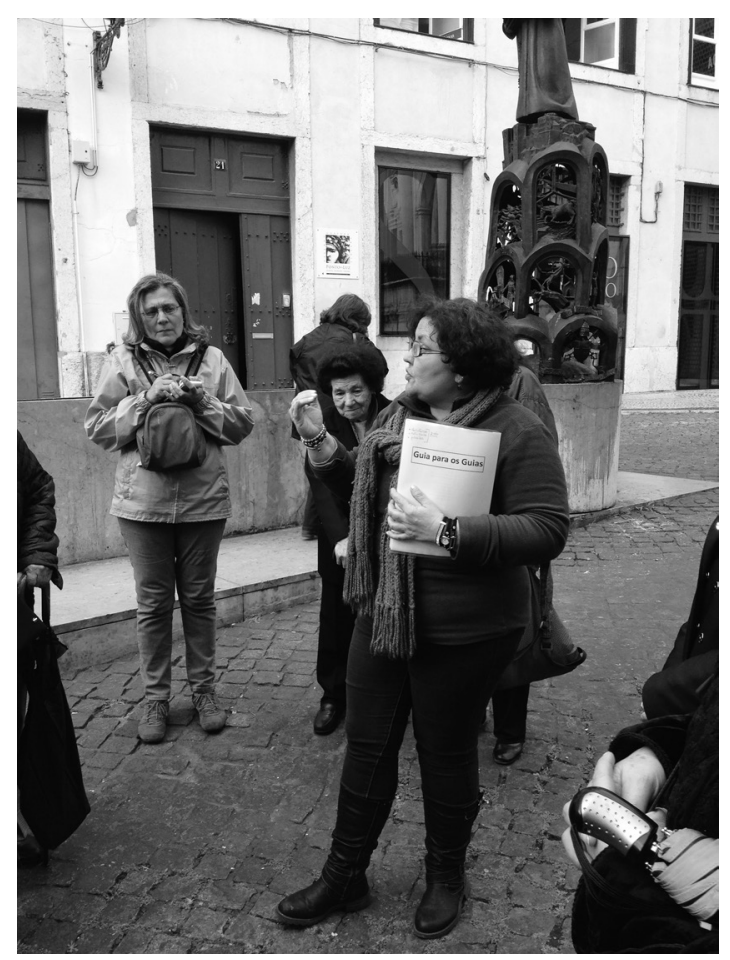

Figure 3

La guide du service culturel municipal du Musée de Lisbonne, au début d'une visite guidée du Musée de saint Antoine, Lisbonne, 2015

(C) Photographie de l'auteur 
Les prêtres et les guides des groupes de pèlerins qui visitent le sanctuaire prennent de plus une part active dans la narration. Selon un relevé statistique du nombre de groupes ayant réservé une visite en 2014, les cinq premières nationalités de pèlerins sont les Italiens (722), les Polonais (215), les Brésiliens (200), les Espagnols (131) et les Portugais (121) $)^{5}$. Les groupes s'arrêtent un jour à Lisbonne pour débuter ou terminer un parcours qui les mène le plus souvent à Fátima, SaintJacques-de-Compostelle, Lourdes, Rome ou Jérusalem. Ils visitent le centre-ville et le quartier d'Alfama, où se situent le sanctuaire, la cathédrale, plusieurs points de vue panoramiques et les restaurants qui offrent des spectacles de fado. La visite est donc motivée par l'emplacement du lieu, sur un axe touristique majeur de Lisbonne, par l'importance de la figure du saint dans le pays d'origine des pèlerins (notamment pour les Italiens et les Brésiliens) et par la possibilité, pour leurs prêtres, de pouvoir célébrer une messe. Ces visiteurs religieux sont accompagnés par un guide de l'entreprise de tourisme qui a organisé le voyage, souvent de leur propre nationalité, auquel s'ajoute parfois un guide local. Les sources de connaissance des guides sont très hétérogènes et la personnalité de chacun imprime une coloration particulière à la visite, notamment en ce qui concerne la foi en Dieu. Il est donc assez difficile de dresser un portrait type de ces guides. Ils donnent parfois une importance centrale à l'histoire du sanctuaire ou au lien entre le saint et la ville. Mais les prêtres qui font partie du groupe contribuent également, pendant la visite et pendant la messe, à la narration de l'hagiographie. Certains pèlerins qui ont, selon l'expression qu'ils utilisent, « une dévotion spéciale à saint Antoine » n'hésitent pas non plus à prendre la parole pour compléter le récit par l'évocation d'une intercession ou d'un miracle personnel. Une discussion peut s'engager également avec les Franciscains présents, et le passage par le magasin du sanctuaire ajoute une expérience visuelle et écrite d'Antoine, par l'achat des statuettes, des images pieuses ou des publications.

5. Voz de santo António, Année XLI, 1, 2014, p. 7. 
104 Des vies extraordinaires : les territoires du récit $-\mathrm{n}^{\circ} 79$

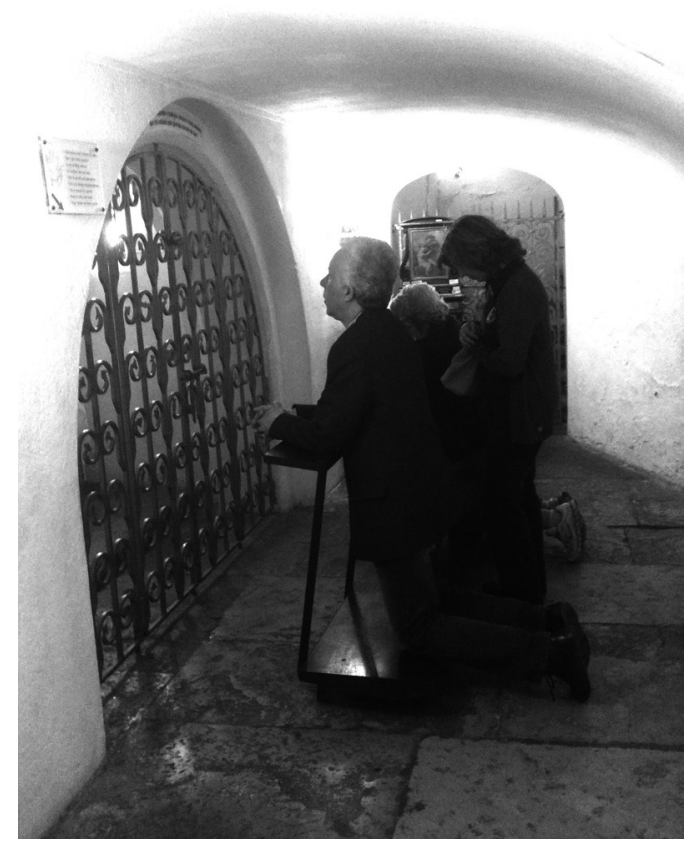

Figure 4

Fidèles en prière dans la chambre supposée de naissance de saint Antoine, église maison de saint Antoine, Lisbonne, 2014

(c) Photographie de l'auteur

Enfin, le dernier ensemble de narrateurs se compose des guides de groupes touristes qui ne participent pas à un pèlerinage, mais à une visite classique de la ville. Ces groupes sont le plus souvent clients d'agences étrangères ou locales, ou des compagnies de croisière qui font escale à Lisbonne. Il peut s'agir de groupes portugais, d'amis ou d'employés d'entreprise en séminaire, qui ponctuellement font appel à un guide. Là encore, le passage par le sanctuaire est principalement dû à l'emplacement du lieu, sur le chemin qui mène du centre-ville à Alfama. Les guides de ces groupes sont souvent locaux et le motif hagiographique qui est le plus souvent mis en avant est celui de l'《authenticité » et de la « popularité » du culte d'Antoine à Lisbonne. Le saint fait ici figure d'emblème culturel en représentant un aspect de la culture locale. L'hagiographie proprement dite et la dimension religieuse n'apparaissent pas dans ce cas comme des éléments centraux du discours, cependant, les guides évoquent des épisodes miraculeux de sa vie, et 
l'on voit facilement des touristes prier (figure 4) et faire une offrande après avoir entendu le guide et pris des photographies du lieu.

\section{Localiser saint Antoine à Lisbonne}

La multiplicité des statuts des narrateurs et des modalités de performance du récit de sa vie ne doit pas cacher une représentation partagée et un désir commun de faire dialoguer la reconnaissance universelle de saint Antoine et sa qualité de saint portugais.

L'argument principal qui est mobilisé repose sur une citation du pape Léon XIII en 1880 : « saint Antoine n'est pas seulement le saint de Padoue, mais le saint du monde entier $\gg^{6}$. La citation est transcrite en grandes lettres dans la salle du musée consacrée à l'iconographie et on l'entend prononcée par les guides et les Franciscains de manière récurrente. Chaque acteur tente ainsi de relativiser l'appartenance padouane de saint Antoine, en le rattachant plutôt « au monde entier », et par conséquent à Lisbonne, le lieu de sa naissance.

La tendance n'est pas récente et s'inscrit dans la longue histoire de la revendication politique de l'appartenance d'Antoine au Portugal, comme on l'a vu plus haut. On peut ainsi retrouver dans les recueils de traditions populaires (notamment Gomes Teixeira, 1931 ; Mattos, 1937) des mentions explicites de revendication de l'origine portugaise du saint, comme le poème écrit sur un plat de céramique de Coimbra du début du $\mathrm{xx}^{\mathrm{e}}$ siècle figurant dans la collection du musée : «Qui dit qu'Antoine est de Padoue/N'est pas un bon Portugais : /Le saint est de Lisbonne/Que cela soit dit une bonne fois pour toutes $! \gg^{7}$

Ces revendications d'attachement d'Antoine à Lisbonne se transposent sans ambiguïté dans les discours contemporains des prêtres ou des guides. La première information transmise aux visiteurs est celle de l'identification du sanctuaire avec le lieu de naissance d'Antoine. Les guides débutent systématiquement la visite de l'église en disant que le saint de Padoue est né à Lisbonne et que les Portugais le connaissent sous le nom de saint Antoine de Lisbonne. Les visiteurs non accom-

6. Cité in Santos (2014, p. 162). Il est difficile de trouver la trace officielle de cette expression qui revient souvent dans les discours contemporains. Il s'agirait d'un extrait d'une réponse que le pape aurait donné au fondateur d'une association de dévots de Padoue. Voir http://www.santodeimiracoli.org/content/associazione-universale-di-santantonio, consultée le 13 mai 2013.

7. "Quem diz António de Pádua/Nunca foi bom Português:/O santinho é de Lisboa,/ Fique assente de uma vez!". Musée de Lisbonne, Pôle de Saint-Antoine, exposition permanente, Usine Veuve Alfredo Oliveira, Coimbra, $x^{e}{ }^{e}$ siècle, inv. MA.CER.17. 
pagnés peuvent lire ce même message sur les murs du porche de la basilique (figure 5). Au Portugal, Padoue, le lieu de la mort du saint, devient ainsi totalement secondaire dans le processus de localisation. En suivant la même logique, les prêtres franciscains n'oublient jamais, au moment de l'introduction de la messe, de l'invocation des saints et parfois dans leurs sermons, de citer le saint du lieu selon une formule fixe : «saint Antoine qui est né ici ${ }^{8}$. Mais la construction d'une origine portugaise du saint n'est pas le fait des seuls Portugais. Un prêtre anglais, qui accompagnait un groupe de pèlerins de sa paroisse fin juin 2014, donnait sa propre version : « [si] les Italiens l'ont choisi comme leur propre patron, il est en fait né à Lisbonne. Et nous sommes donc aujourd'hui dans les lieux qui l'ont vu naître, grandir, puis devenir un homme, un prêtre et un grand théologien ».

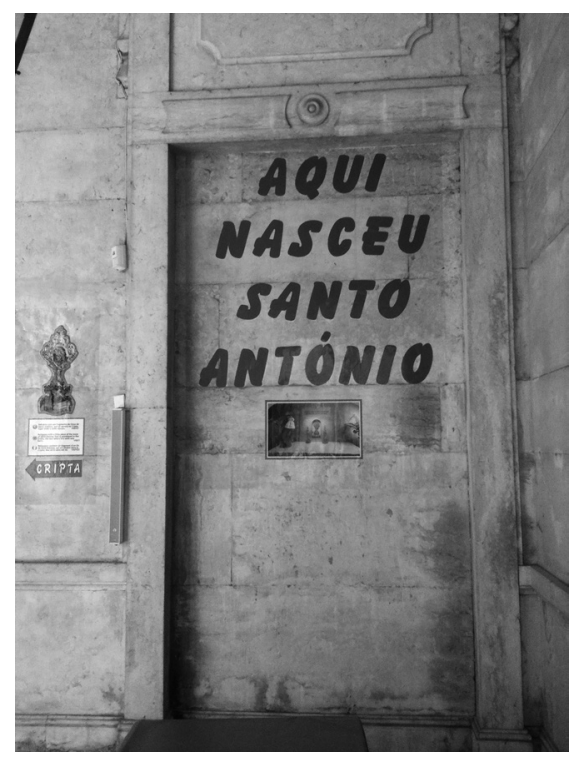

Figure 5

«Ici est né saint Antoine », message d'identification du lieu de naissance de saint Antoine, église-maison de saint Antoine, Lisbonne, 2014

(C) Photographie de l'auteur

Les techniques rhétoriques de renversement du lieu identificatoire du saint constituent une des stratégies principales de la qualification portugaise d'Antoine, à l'opposé de ce qui est plus classiquement le cas sur les tombeaux sacrés dans les mondes catholiques, musulmans et juifs (Kerrou, 1999 ; Amri et Gril, 2008). Mais elles ne sont pas pour autant profondément bouleversées. Au contraire, qu'il s'agisse du lieu de naissance d'Antoine ou de la mort d'un autre personnage, se 
mettent en place des fonctionnements similaires de localisation des figures saintes (Claverie, 2003 ; Albert-Llorca, 2002 ; Isnart, 2008). À Lisbonne comme ailleurs, ces techniques narratives font intervenir le nom et les caractéristiques du lieu dans les discours des prêtres ou des guides, afin d'assurer la concordance entre le récit de la vie du saint et le lieu de son culte.

\section{Un saint du peuple}

Au-delà de cette localisation narrative, les différents acteurs insistent sur le culte du saint au Portugal, qui semble lui donner une identité particulière par rapport à d'autres contextes nationaux et le définir comme un être spécifique. Pour les guides locaux, pour les personnels du musée, et en partie pour les Franciscains, saint Antoine de Lisbonne est «um santo popular » ou «um santo do povo », un saint populaire ou un saint du peuple?

La catégorie de « santo popular » désigne de manière courante et officielle, dans la vie festive et religieuse portugaise, une série de saints honorés au mois de juin lors des fêtes municipales de plusieurs grandes villes du pays ${ }^{10}$. Liant foire marchande, divertissement forain, commensalité, dévotions religieuses et présence des hommes politiques, on compte dans cette catégorie principalement les fêtes de saint Antoine de Lisbonne (13 juin), de saint Jean Baptiste (24 juin) et de saint Pierre (29 juin). L'ethnographie classique a donné une grande place à ces rituels dans l'autoportrait ethnologique du Portugal, en décrivant les différents aspects de ces fêtes (par exemple : Chaves, 1938). La filmographie de fiction des années 1930 et 1940, ainsi que les films de promotion touristique, qui ont pu occasionnellement servir de support de propagande culturelle de l'identité nationale, ne manquent pas de mettre en scène les fêtes des saints populaires, comme dans $O$ pátio das cantigas (Ribeiro, 1941), dont l'action se situe pendant les fêtes de Lisbonne.

Pour expliquer le caractère populaire de ces saints, on retrouve dans la littérature ethnographique, depuis la fin du $\mathrm{XIX}^{\mathrm{e}}$ siècle jusqu'à aujourd'hui, une

9. La notion de povo a été mobilisée de différentes façons par les pouvoirs politiques portugais, notamment par l'Estado Novo et les mouvements de démocratisation postrévolutionnaire (sur ces points, Marques Alves, 2010). Je travaille ici sur les connotations du mot qui ont cours aujourd'hui parmi les narrateurs de la vie de saint Antoine, sans détailler celles des périodes historiques précédentes.

10. Ce cycle de juin, on le verra, doit être rapproché des caractéristiques qu'énumère Van Gennep pour le cycle de saint Jean (1999 [1949], p. 1427-1740); pour une sociologie contemporaine des fêtes de saint Antoine à Lisbonne, voir FrúGOLI, 2014. 
interprétation évolutionniste de certains éléments des festivités, faisant référence à la théorie des survivances des cultes païens dans la culture contemporaine. Généralement, les auteurs rapprochent les relations amoureuses exacerbées par les festivités nocturnes et la musique, les bûchers, les danses et les pratiques magiques des cultes préchrétiens du soleil, de la fertilité et de la sexualité.

Dans le musée, la section dédiée aux manifestations populaires du culte de saint Antoine expose une série d'objets illustrant cette perspective. Ex-voto, pains distribués pendant la fête, manjerico (le pot de basilic avec œillet de papier et poésie amoureuse) (figure 6), tronos (les autels éphémères montés par les familles devant leurs portes), ou marchas (les défilés costumés et concours entre groupes de quartier) soulignent les thématiques de la recherche populaire de la guérison, des partenaires sexuels ou de l'esprit collectif. Ces objets et ces rituels relieraient les rites du présent et le fonds magico-religieux ancien. Lors des visites guidées du musée, l'une des employées du service municipal de la culture se prête à une lecture exemplaire du manjerico. Il s'agit d'un pot de basilic, orné d'un œillet et d'une courte poésie de courtisement, offert traditionnellement à l'être aimé. Le manjerico est par ailleurs communément reconnu comme un symbole des fêtes des saints populaires au Portugal : il est présent sur les tables de restaurants, sur les affiches de la fête et sur les étals des fleuristes et des vendeurs ambulants pendant les festivités. La guide rappelle d'abord le rôle du basilic dans les relations amoureuses et ce geste anodin, passer les paumes des mains pour sentir l'odeur caractéristique de la plante, que tous les Portugais connaissent et pratiquent. Pour l'employée municipale, le basilic représenterait le pubis de la femme qu'il s'agit de caresser sans brusquerie dans les relations amoureuses. Cette interprétation d'un élément festif, que la guide présente comme typique du culte populaire de saint Antoine, rappelle un symbole sexuel populaire qui fait écho au rôle de marieur qu'on lui prête. Par là, elle confirme la profondeur symbolique de pratiques populaires venues d'universaux préchrétiens, elle explique l'attachement populaire au saint, en appliquant la théorie de la survivance de motifs archaïques et sexuels dans la tradition populaire portugaise contemporaine, et elle nationalise le rituel de saint Antoine et le motif du basilic ${ }^{11}$.

11. Il n'est pas étonnant de trouver cet usage amoureux du basilic, puisque en France (VAn Gennep, 1999, p. 1608-1640) comme dans le bassin méditerranéen (Belmont, 2003), le basilic comme les herbes de la Saint-Jean sont étroitement liés aux pratiques de courtisement et de mariage. Nicole Belmont rappelle les parallèles rituels du conte-type T879 la Fille au pot de basilic et interprète cet ensemble comme le passage du statut de jeune fille épousable à la femme mariée. 
Les frères franciscains réfutent bien évidemment cette interprétation évolutionniste et les éléments profanes du culte, comme les bals et la consommation d'alcool et de sardines grillées, qui sont associés aux fêtes de Lisbonne. En suivant la tradition des usages classiques de la sainteté, les sermons des Franciscains rappellent que saint Antoine n'est pas seulement un recours dans des moments difficiles ou le simple prétexte à des fêtes populaires. Il doit être pris pour modèle d'un comportement exemplaire pour l'ensemble du peuple chrétien afin de fortifier la foi en Dieu. Mais ils admettent, de manière plus générale, le pouvoir de médiation recherché par les fidèles et leurs marques de reconnaissance comme des signes de la popularité du saint parmi le « peuple ». Les demandes qui sont adressées au saint relèvent bien pour les Franciscains de besoins élémentaires et universels - y compris celui du mariage, il est bon et dans l'ordre des choses de s'en remettre à saint Antoine, « le grand ami et compagnon du peuple de Lisbonne » comme le définit dans ses sermons le recteur du sanctuaire. Ainsi, l'argument de la « popularité » du saint, qu'il provienne d'une application de la théorie de la survivance ou d'une lecture en terme de modèle de sainteté et de pouvoir d'intercession, contribue à construire l'image d'un saint Antoine en adéquation avec son origine lisboète ${ }^{12}$.

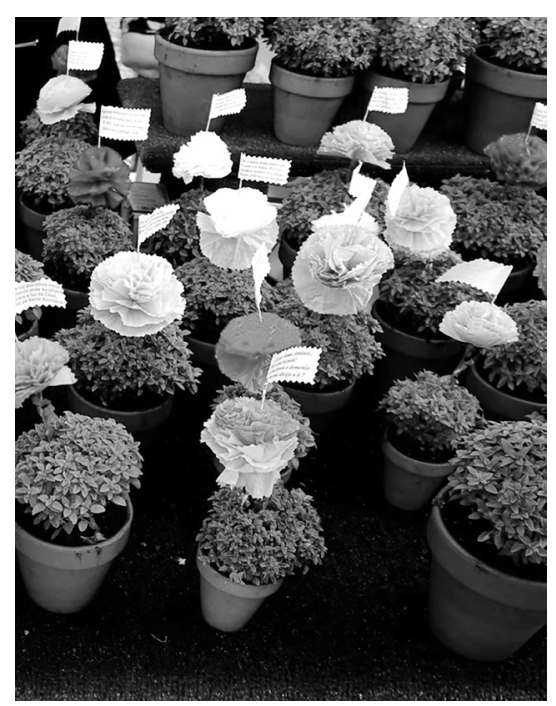

Figure 6 : Étal d'une vendeuse de manjerico, Lisbonne, 2014

(C) Photographie de l'auteur

12. Au risque de passer sous silence le fait que la fonction de saint populaire, de saint marieur et d'auxiliaire pour retrouver les choses perdues se retrouve dans bien d'autres contextes, notamment au Brésil et en Europe du Sud (Menezes, 2004, p. 158-163). 
Mais la «popularité » du saint peut encore revêtir un autre sens, celui de la familiarité des rapports entre lui et ses dévots, qui se démarque de l'argument évolutionniste et la rhétorique du saint modèle. En même temps que la construction de l'ascendance païenne du culte de saint Antoine permet d'en révéler les supposées modalités typiquement portugaises, les guides et les prêtres mettent en avant l'intimité que les fidèles entretiennent avec le saint. Par exemple, le musée expose une série de statuettes domestiques, fabriquées en grand nombre et présentes dans de nombreux foyers, qui témoignent de la diffusion sociale du culte. On signale aux visiteurs que, selon la littérature ethnographique, les statues pouvaient subir des humiliations (en tournant le visage vers le mur ou en privant le saint de l'Enfant-Jésus), désignant ainsi un rapport familier entre le saint et ses dévots ${ }^{13}$. Le frère chargé de l'accueil au sanctuaire m'a souvent répété qu'il restait toujours surpris du nombre de personnes qui se déclaraient « dévots de saint Antoine », de toutes conditions et nationalités, touchant ainsi la preuve d'une popularité étonnante de son saint. De leur côté, les guides locaux, accueillant des visiteurs étrangers, présentent saint Antoine comme un recours courant et intime des Portugais. L'un d'eux introduit ainsi la visite de la chambre natale : « Quand nous avons un problème, nous les Portugais, nous venons toujours à saint Antoine, nous lui demandons toujours quelque chose. C'est notre saint qui nous aide. Et spécialement les jeunes filles qui cherchent un mari (rires) ».

L'inscription du culte dans la théorie de la survivance (un cycle rituel particulier, lié à la fertilité et à la fréquentation des filles et des garçons), et son association multidimensionnelle avec le « peuple » donnent au saint, selon les narrateurs de sa vie et les acteurs de son culte, une particularité religieuse et culturelle qui est intimement liée à son appartenance portugaise. En associant une hagiographie localisée à des qualifications supposées spécifiques et locales du culte, les différents narrateurs entretiennent l'appartenance du saint au lieu et son lien avec ses fidèles.

\section{Les effets ambivalents des discours}

Parmi les conséquences des multiples modalités des narrations de la vie de saint Antoine que l'on peut tenter de mettre au jour, deux me paraissent centrales.

13. On connait, dans le répertoire des dévotions rituelles envers les saints, de telles pratiques d'humiliation de la statue, dont les folkloristes ont donné de nombreux exemples (par exemple, SÉвILLот, 1905, p. 376-377), ainsi que les menaces familières que les dévots français adressent à saint Antoine de Padoue lorsqu' ils veulent retrouver une chose perdue («Saint Antoine de Padoue/Grand voleur et grand filou/Qui voyez dans tous les petits trous/Rendez ce qui n'est pas à vous. » VAUGEOIS, 1909, p. 138). 
En premier lieu, l'insistance sur son origine portugaise et sur son culte populaire tend à effacer la figure du docteur de l'Église catholique, érudit, théologien, dirigeant de son ordre, d'origine noble et proche du pouvoir. Elle connote plutôt un saint aimé du peuple, répondant à des désirs simples et vivant auprès de toutes les catégories sociales. De nombreux guides évoquent la naissance dans une famille de petite noblesse et l'éducation quasi aristocratique du jeune Antoine ainsi que ses succès dans les milieux intellectuels et dans la hiérarchie de son ordre. Mais ils placent volontiers l'expression de sa maturité en Italie et en France et préfèrent mettre en avant la popularité du saint à Lisbonne. Les Franciscains offrent, parfois pendant leurs sermons, des commentaires sur les œuvres écrites de leur saint, ils relatent également le soutien de différents souverains portugais à l'entretien, la décoration et la vie du sanctuaire, mais rappellent dans le même temps la présence du saint parmi le peuple de Lisbonne, ce dont témoignent, selon eux, les billets votifs, les offrandes déposés près des images ou la foule qui se presse lors de la procession annuelle. Ce balancement entre les deux représentations du saint peut contribuer à la dynamique de distinction entre différents groupes sociaux, les religieux préférant le docteur de l'Église, les fidèles le saint familier que l'on peut facilement rudoyer. Robert Hertz avait pu analyser une telle distinction pour saint Besse, entre l'évêque des clercs de la plaine et le berger-soldat des paysans de la montagne (Hertz, 2014 [1913]). À Lisbonne, l'utilisation et les connotations des termes « peuple » et « populaire » pour qualifier le culte d'Antoine renvoient bien évidemment à une distinction sociale de cet ordre, entre gens ordinaires et élite religieuse et politique. Avec une nuance cependant : les connotations et les fonctions semblent balancer entre le savant théologien et l'intercesseur du peuple pour une seule et même représentation de saint Antoine. Il ne s'agit donc pas à proprement parler du dédoublement d'une seule personnalité en deux figures surnaturelles comme saint Besse. À Lisbonne, nous avons plutôt à faire au choix de privilégier la figure populaire d'Antoine, sans que les narrateurs s'empêchent d'activer, selon les moments rituels, les publics en présence et les arguments à valoriser, l'une et/ou l'autre des facettes de sa personnalité. La pluralité des aspects valorisés de la vie permet d'accéder ainsi à des usages différenciés d'une même personne surnaturelle, tour à tour support d'une politique de construction et d'entretien du nationalisme, emblème de la culture populaire locale ou modèle de comportement chrétien.

Le second effet des énonciations multiples de l'hagiographie réside dans un brouillage des usages religieux et des usages patrimoniaux du saint dans les mécanismes de transmission culturelle. D'un côté, l'anthropologie et la sociologie des religions ont très tôt mis en avant la question de la transmission des valeurs et des pratiques religieuses. Depuis l'échelle collective jusqu'au niveau de l'individu, 
les conditions d'apprentissage et de transfert entre générations mobilisent des concepts comme celui de mémoire et d'identité collectives (Halbwachs, 1950 ; Assmann, 2006), de temps et de cycle (Van Gennep, 1998-1999; Hubert, 1904) ou de transmission et d'éducation (Hérault, 1997 ; Berliner et Sarró, 2007). D’un autre côté, l'institution du patrimoine culturel comme image et idéal collectif repose sur des principes de passage entre générations à travers des dispositifs comme le monument, le musée, la visite guidée ou l'éducation formelle au patrimoine (Poulot, 2006 ; Smith, 2006). Au sein de l'Église catholique, mais pas seulement, les enjeux de transmission patrimoniale sont centraux et ouvrent l'activité religieuse proprement dite à l'intégration de l'action culturelle et touristique dans ses propres cadres de performance (Bennet, 2009 ; Saint-Martin, 2013 ; Isnart, 2014). Le but de ces actions n'est pas principalement la conversion des noncroyants, mais la transmission de connaissances historiques sur le fait religieux et les sentiments spirituels (Badone, 2015, Sagnes, 2014).

Le cas de saint Antoine à Lisbonne apparaît exemplaire de ce recouvrement des champs de l'institution de la culture et de la transmission de la religion. Le récit hagiographique n'est plus seulement transmis par les clercs, comme cela pouvait être le cas pendant le Moyen Âge alors que la prédication prenait une place plus grande dans les processus d'évangélisation et donnait à l'exemplum et aux motifs hagiographiques un rôle central dans la rhétorique (Schmitt, 2005). Au-delà des Franciscains du sanctuaire et des prêtres des groupes de pèlerins, ceux qui racontent la vie du saint de Lisbonne sont des laïcs, guides touristiques ou employés municipaux, qui transmettent des éléments d'histoire, d'histoire de l'art et d'histoire religieuse, sans se passer de diffuser les récits de miracles, les épopées des reliques, ou la chronologie des lieux de culte. Il n'y a donc pas de séparation nette entre un champ de la culture, supposément rationnel et érudit, et un champ de la religion, tourné vers la spiritualité et la croyance. La légitimité de l'énonciation hagiographique semble ainsi aujourd'hui plus négociée et partagée entre les narrateurs. Le frère responsable des visites du sanctuaire organise d'ailleurs chaque année une formation pour les guides touristiques de Lisbonne, durant laquelle il rappelle la dimension spirituelle du lieu et de son saint patron, les qualifiant explicitement d'objets de croyances religieuses. Il invite de plus les responsables de l'association professionnelle des guides à assister à l'une des messes de la fête en leur donnant le rôle de « parrain de saint Antoine ». Il leur attribue ainsi un rôle religieux et rituel tout en légitimant leur fonction patrimoniale de transmission. Le directeur du musée municipal de saint Antoine, anthropologue de formation, manifeste la même ambivalence en construisant, avec le recteur du sanctuaire, un dispositif de relance de la tradition des tronos (figure 7), les autels éphémères qui décorent les rues et les boutiques de la ville, et en créant une page Facebook 
intitulée Património Santo António. Là encore, il est peu aisé de déterminer une frontière nette entre l'activité de transmission religieuse d'une pratique rituelle et une pratique d'animation culturelle de type patrimonial.

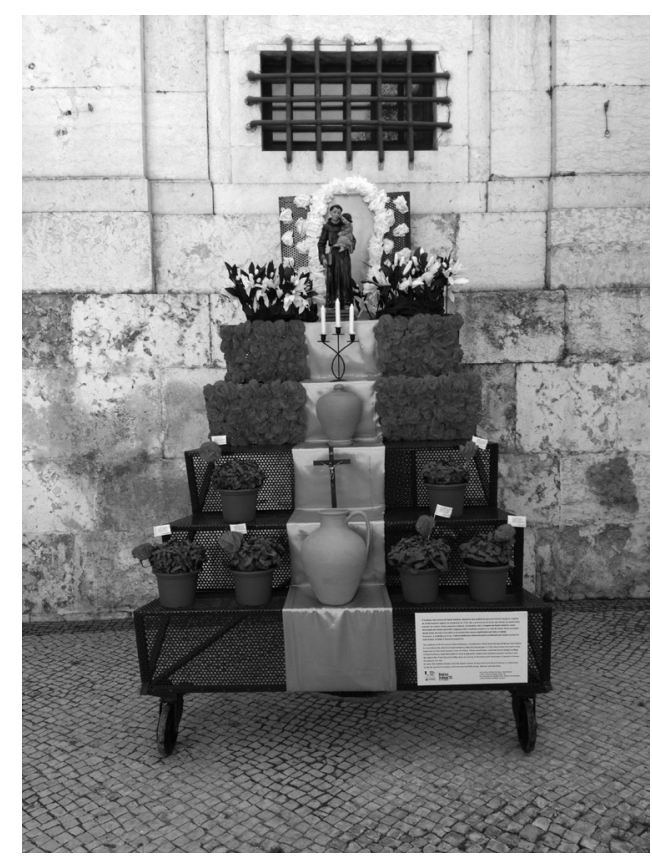

Figure 7

Trono de santo António, réalisé par l'équipe du Musée de saint Antoine dans le cadre d'une action de revitalisation de la tradition des tronos, Lisbonne, 2015

(C) Photographie de l'auteur

\section{Conclusion}

La description des modalités contemporaines de la narration de la vie de saint Antoine à Lisbonne a permis de souligner la diversité des statuts des narrateurs, des motivations de leurs performances et des effets de leurs discours. Les liens que les prêtres, les guides touristiques et les professionnels de la culture tissent avec le saint et entretiennent entre eux semblent confirmer le poids des usages politiques et culturels du fait religieux et du rituel, qui a été déjà repéré et analysé par les sciences sociales, notamment par l'anthropologie des cultures traditionnelles européennes (Centlivres, Fabre et Zonabend, 1999 ; Hobsbawm et Ranger, 2006 ; Boissevain, 1992) ou les études du tourisme (Boissevain, 2010 ; Leite, 2005). 
Mais l'ethnographie des visites au sanctuaire de Lisbonne nous incite cependant à poser de nouvelles questions, en examinant les effets de ces usages sur le religieux lui-même, et non sur la société ou les dynamiques politiques. Ce travail pourrait ainsi contribuer à une meilleure compréhension des narrations hagiographiques contemporaines en soulignant la pluralité des acteurs et des valeurs en jeu dans les visites de sites religieux aujourd'hui.

L'anthropologie du tourisme et des pèlerinages a souvent favorisé l'expérience des touristes et des pèlerins en tant qu'individus en situation de déplacement culturel ou de rencontre spirituelle (Graburn, 1977). Les acteurs locaux qui reçoivent ces visiteurs, les guides, les hôteliers, les spécialistes religieux ont été moins souvent pris en compte dans les analyses et leur sociologie, comme la description de leurs discours, ont souvent été mises de côté (Salazar, 2006 ; Sampaio, Simoni et Isnart, 2014). Il ressort de l'étude du cas de saint Antoine à Lisbonne que les intérêts des acteurs religieux, patrimoniaux et touristiques se conjuguent à des qualifications ambivalentes du saint, de son histoire et de son lieu et superposent les différentes motivations des acteurs en présence. En essayant d'adopter le point de vue des énonciateurs contemporains de l'hagiographie, il devient difficile de séparer et de hiérarchiser les motivations de chacun de ces narrateurs et les conséquences de leurs performances. Tous ces acteurs s'inscrivent plutôt dans un continuum entre des usages religieux et des usages sociaux de la sainteté. Cependant, la diversité des locuteurs donne toujours sens localement à la figure du saint et lui procure paradoxalement une certaine stabilité religieuse, culturelle et politique. Cette étude concourt ainsi à révéler la spécificité contemporaine de la narration de la vie des saints, notamment en contexte catholique européen. Les clercs, locaux ou étrangers, les guides humains liés au tourisme culturel ou religieux et les personnels des musées à contenu religieux, partagent aujourd'hui la tâche de transmettre des objets religieux, selon des modalités et dans des dispositifs qui leur sont propres, l'objet de ces performances restant pourtant le même. La narration hagiographique contemporaine, conjuguant finement des façons de dire le saint et des manières de transmettre la culture religieuse révèle alors la porosité des frontières entre religion, tourisme et patrimoine et conduit à penser ces trois domaines comme un champ continu et fractionné à la fois.

\section{Bibliographie}

Albera, Dionigi et Fliche, Benoît, 2012, « Muslim Devotional Practices in Christian Shrines: The Case of Istanbul », in Albera Dionigi et Maria (dir.), Sharing sacred spaces in the Mediterranean. Christians, Muslims, and Jews at Shrines and Sanctuaries, Bloomington: Indiana University Press. 
Albert-Llorca, Marlène, 2002, les Vierges miraculeuses. Légendes et rituels, Paris : Gallimard, « Le Temps des images ».

Amri, Nelly et Gril, Denis (dir.), 2008, Saint et Sainteté dans le christianisme et l'islam. Le regard des sciences de l'homme, Paris : Maisonneuve \& Larose, Aixen-Provence : Maison méditerranéenne des sciences de l'homme.

Assmann, Jan, 2006, Religion and Cultural Memory, Stanford: Stanford University Press.

Badone, Ellen et Roseman, Sharon R. (dir.), 2004, Intersecting Journeys. The Anthropology of Pilgrimage and Tourism, Urbana \& Chicago: University of Illinois Press.

BADONE, Ellen, 2015, "Religious Heritage and the Re-enchantment of the World in Brittany”, Material religion, vol. 11, no. 1.

Belmont, Nicole, 2003, « le Langage du basilic. À propos du conte-nouvelle la Fille au pot de basilic », Cabiers de littérature orale, $\mathrm{n}^{\circ}$ 53-54.

Bennet, Oliver, 2009, "On Religion and Cultural Policy. Notes on the Roman Catholic Church”, International Journal of Cultural Policy, vol. 15, no. 2.

Berliner, David et SArró, Ramon(dir.),2007, LearningReligion. Anthroplogical Approaches, London: Berghahn.

Boissevain, Jeremy (dir.), 1992, Revitalizing European Rituals, London: Routledge.

Boissevain, Katia (dir.), 2010, Maghreb et sciences sociales, dossier « Nouveaux usages touristiques de la culture religieuse », Tunis : IRMC.

Bollandistes, 1643, Acta sanctorum, ianuarii, II, Anvers : Thieullier.

Bollandistes, 1688, Acta sanctorum, junii, II, Anvers : Thieullier.

Brévot Dromzée, Claude, 2005, «'Cher saint Antoine...' », Communication, vol. $23, \mathrm{n}^{\circ} 2$. 
Centlivres, Pierre, Fabre, Daniel, Zonabend, Françoise (dir.), 1999, la Fabrique des héros, Paris : Éditions de la Maison des sciences de l'homme.

Chaves, Luís, 1938, « Os santos populares. Santo António e São Pedro nas tradições portuguesas », Brotéria. Revista contemporânea de cultura, ${ }^{\circ} 17$.

Claverie, Élisabeth, 2003, les Guerres de la Vierge. Une anthropologie des apparitions, Paris : Gallimard.

Czarnoski, Stefan, 1919, le Culte du héros et ses conditions sociales. Saint Patrick, héros national de l'Irlande, Paris: Alcan.

Eade, John et Sallnow, Michael (dir.), 1991, Contesting the Sacred. The anthropology of Christian pilgrimage, London et New York: Routledge.

Flatrauer, Claude, et al. (dir.), 2012, Patrimoine religieux. Désacralisation, requalification, réappropriation, Paris : Riveneuve.

FRÚ GOLI, Heitor Jr., 2014, « Festas populares em Lisboa. Uma etnografia a partir do Bairro Alto », Etnográfica, vol. 18, nº 1 .

$\mathrm{GEPB}=$ Grande enciclopédia portuguesa e brasileira, Lisbonne et Rio de Janeiro : Enciclopédia, 1936-1960.

Gomes Teixeira, Francisco, 1931, Santo António de Lisboa. Historia, tradição e lenda, Lisbonne : Livraria Clássica.

Graburn, Nelson, 1977, “Tourism. The Sacred Journey”, in Smith Valene L. (dir.), Hosts and Guests. The Anthropology of Tourism, Oxford: Basil Blackwell.

Halbwachs, Maurice, 1950, la Mémoire collective, Paris : Presses universitaires de France.

HÉRAUlT, Laurence, 1997, « Apprendre à communier », Journal des anthropologues, $\mathrm{n}^{\circ} 71$, https://jda.revues.org/2526.

Hertz, Robert, 2014, OEuvres publiées, Paris : Classiques Garnier. 
Новsbawm, Eric et Ranger, Terence (dir.), 2006 [1983], l'Invention des traditions, Paris : Éditions Amsterdam.

Hubert, Henri, 1904, «Étude sommaire de la représentation du temps dans la religion et la magie », Rapport sommaire sur les conférences de l'exercice 1904-1905.

Hubert, Henri, 1919, « Préface », in Czarnowski, Stefan, le Culte des héros et ses conditions sociales. Saint Patrick, héros national de l'Irlande, Paris : Félix Alcan.

Isnart, Cyril, 2008, Saints légionnaires des Alpes du Sud. Ethnologie d'une sainteté locale, Paris : Éditions de la Maison des sciences de l'homme.

Isnart, Cyril, 2009, « Patrimonialiser son culte. Activités rituelles et effets patrimoniaux dans une confrérie catholique des Alpes », in BERTOHOLD Étienne et Dormaels Mathieu (dir.), Patrimoine et sacralisation. Patrimonialisation du sacré, Québec : Éditions. Multimondes.

Isnart, Cyril, 2014, "Changing the Face of Catholicism in a Tourist Context. Ritual Dynamic, Heritage Care and the Rhetoric of Tourism Transformation in a Religious Minority", Journal of Tourism and Cultural Change, vol. 12, no 2 .

Kerrou, Mohamed (dir.), 1998, l'Autorité des saints. Perspectives historiques et socio-anthropologiques en Méditerranée occidentale, Paris : Éditions Recherche sur les civilisations.

LE Goff, Jacques, 1999, Saint François d'Assise, Paris : Folio.

Leite, Naomi, 2005, "Travels to an Ancestral Past. On Diasporic Tourism, Embodied Memory, and Identity”, Antropológicas, no. 9.

Marques Alves, Vera, 2010, « O povo do Estado Novo », in Neves José (dir.), Como se faz um Povo, Lisbonne: EDP/Tinta da China.

Mattos, Armando de, 1937, Santo António na tradição popular (subsidio etnográfico), Porto : Livraria Civilização. 
CAHIERS DE LITTÉRATURE ORALE

118 Des vies extraordinaires : les territoires du récit $-\mathrm{n}^{\circ} 79$

Menezes, Renata de Castro, 2004, A dinâmica do sagrado. Rituais, sociabilidade e santidade num convento do Rio de Janeiro, Rio de Janeiro : Relume-Dumará.

Pacheco, Milton Pedor Dias, 2009, « Os proto-mártires de Marrocos da Ordem de São Francisco », Revista lusófona de ciência das religiōes, vol. VIII, nº 15.

Paine, Crispin, 2013, Religious Objects in Museums. Private Lives and Public Duties, London: Bloomsbury Academic.

Pereira, Pedro Teotónio, 2015, « Santo António, protetor da cidade de Lisboa », Rossio. Estudos de Lisboa, $\mathrm{n}^{\circ} 5$.

Pestre de Almeida, Lilian, 2009, « Des captifs et des martyrs au Maroc. Étude d'un thème iconographique franciscain : les Martyrs du Maroc, son instrumentalisation et sa diffusion en Europe et en Amérique », Rivista dell'Istituto di Storia dell'Europa Mediterranea, $\mathrm{n}^{\circ} 3$.

Pinto, Augusto, 1942, O valor turístico de Santo António de Lisboa, Lisbonne : Publicações culturais da Câmara Municipal de Lisboa.

Poulot, Dominique, 2006, Une Histoire du patrimoine en Occident, Paris : Presses Universitaires de France.

Ribeiro, Francisco, 1941, O pátio das cantigas, 101 minutes.

Roger, François, 1971, « Le culte de saint Antoine de Padoue aux Hauts-Buttés (Ardennes). Un siècle d'ex-voto (1871-1971) », Revue historique ardennaise, $\mathrm{n}^{\circ} 5$.

SAGNES, Sylvie, 2014, « Les visites à Notre-Dame. Enjeux et acteurs de la médiation patrimoniale », Séminaire Dynamiques de patrimonialisation des sites et des rituels religieux, EHESS, Paris, 6 février 2014.

SAINT-Martin, Isabelle, 2013, « Regards esthètes et regards croyants. Patrimoine religieux et pastorale du tourisme », in Flatrauer, Claude et al. (dir.), Patrimoine religieux. Désacralisation, requalification, réappropriation, Paris : Riveneuve. 
Salazar, Noel B., 2006, “Touristifying Tanzania. Global Guides, Local Discourses", Annals of Tourism Research, vol. 33, no. 3.

Sampaio, Sofia, Simoni, Valerio, Isnart, Cyril, 2014, "Tourism and Transformation. Negotiating Metaphors, Experiencing Change”, Journal of Tourism and Cultural Change, vol. 12, no. 2.

Santos, Isabel Maria dos, 2014, Do altar ao palco. Santo António na tradição literária, artística e teatral em Portugal e em Espanha, Thèse de doctorat en étude de littérature et de culture, Université de Lisbonne, Faculté des Lettres.

Schmitt, Jean-Claude, 2005, « Trente ans de recherche sur les exempla », Les Cahiers du Centre de recherches historiques. Archives, $\mathrm{n}^{\circ} 35$, mis en ligne le $1 \mathrm{er}$ juin 2011, consulté le 18 juin 2015. URL : http://ccrh.revues.org/3010 ; DOI : $10.4000 /$ ccrh.3010.

SÉbastia, Brigitte, 2004, les Rondes de saint Antoine. Culte, affiction et possession à Puliyampatti (Inde du Sud), Thèse de doctorat en anthropologie, Toulouse, EHESS.

SÉbillot, Paul, 1905, le Folk-lore de France. La mer et les eaux douces, Paris : Guilmoto.

Sequeira, Gustavo de Matos et BRASÃO, Eduardo (dir.), 1940, Mundo português. Imagens de uma exposição histórica, Lisbonne : SNI.

Silva, Maria de Lourdes da, 1991, A reedificação da igreja de Santo António. Espaço, arquitectura, programa 1755-1812, Mémoire de Maîtrise, Histoire de l'art, Université de Lisbonne, Faculté des Lettres.

SMith, Laurajane, 2006, Uses of Heritage, London: Routledge.

Thornton, John, 1998, The Kongolese Saint Anthony: Dona Beatriz Kimpa Vita and the Antonian Movement, 1684-1706, Cambridge: Cambridge University Press.

VAN DiJKN, Willibrord-Christian, 1980, « État actuel du culte à saint Antoine de Padoue dans les églises paroissiales de Paris », in I volti antichi e attuali del santo di Padova, atti del secondo colloquio interdisciplinare l'immagine di s. Antonio, Padova, 9-11 aprile 1979, Padoue : Edizioni Messaggero. 
CAHIERS DE LITTÉRATURE ORALE

120 Des vies extraordinaires : les territoires du récit $-\mathrm{n}^{\circ} 79$

Van Gennep, Arnold, 1998-1999 [1937-1958], le Folklore français, Paris, Robert Laffont. Manuel de folklore français contemporain, Paris : Picard.

Van Gennep,Arnold, 1999 [1949], le Folklore français. 2, Cycles de mai, de la Saint-Jean, de l'été et de l'automne, Paris : Robert Laffont. Manuel de folklore français contemporain. Tome I. IV. Les cérémonies périodiques, cycliques et saisonnières. 2 : Cycle de mai, la Saint-Jean, Paris : Picard.

Vaugeois, Marie-Edmée, 1909, « Traditions et superstitions de la LoireInférieure », Revue des traditions populaires, t. XXIV, $\mathrm{n}^{\circ}$ 4-5.

Résumé : Cet article examine les modalités plurielles de la narration contemporaine de la vie de saint Antoine de Padoue dans le sanctuaire qui est construit à l'emplacement supposé de sa naissance, à Lisbonne au Portugal. En décrivant d'abord la diversité des narrateurs (Franciscains en charge du sanctuaire, guides touristiques, employés des services culturels municipaux et conservateur de musée), on tente de montrer quelles sont les conditions concrètes de la fabrication d'une figure sainte locale, dont les images et le culte sont diffusés de manière universelle. Si la naissance et la formation portugaises du saint apparaissent comme deux motifs narratifs essentiels de localisation, la mise en récit des éléments du rituel populaire au Portugal permet par ailleurs de consolider les liens que le saint entretient avec son lieu. La mobilisation de l'hagiographie portugaise et de certaines pratiques dévotionnelles locales dans les discours de visite du sanctuaire implique d'une part une distinction entre le modèle ecclésiastique, universel et intellectuel de saint Antoine docteur de l'Église et la figure d'intercesseur ordinaire et familier des fidèles portugais. Mais la diversité des acteurs qui transmettent l'hagiographie permet d'autre part d'interroger les superpositions entre les usages religieux, touristiques et patrimoniaux du saint, qui apparaît tout à la fois comme modèle de vie chrétienne, paradigme de la religion populaire locale et expression de l'identité nationale. Le texte contribue ainsi à analyser comment les enjeux du tourisme et de la patrimonialisation se conjuguent intimement aujourd'hui aux pratiques religieuses et imposent une analyse qui prenne en compte la diversité des motivations du fait religieux contemporain.

Mots clefs : saint, récit, tourisme, pèlerinage, Portugal, anthropologie, religion, catholicisme 


\section{Telling the Life of Saint Anthony in Lisbon. Actors, Performances and Effects of Contemporary Hagiographic Discourse}

Abstract: This paper describes and analyses the current modalities of the narrations of the life of the Catholic saint Anthony of Padova, at his so called birthplace in Lisbon (Portugal). Through an ethnography of the visits led by various actors (Franciscan brotherhood, tourist guides, staff of Lisbon's cultural service, head of the museum), we seek to describe the transformation of the universal saint Anthony of Padova into a Portuguese saint. If the biographical sequences of his birth and education in Portugal are classically central in the localisation, the narratives about popular devotions taking place in Lisbon and Portugal also participate in the process. As a consequence, both hagiographic motives and devotional particularities transmitted during the visits seem to erase the ecclesiastical, intellectual and universal figure of Anthony as a Doctor of the Church. Instead, they value the figure of the saint as an ordinary and familiar intercessor of the Portuguese people. On another hand, the diversity of the actors involved also allows us to investigate the overlap of religious, tourist and heritage aims the narrators carry through the different roles of the saint they wish to value (a Christian life model, an example of folk religion, or a symbol of national identity). The text more generally contributes to the understanding of tourism and heritage-making influences on religion, which definitively blur the conditions of today's religious experience.

Keywords: saint, narrative, tourism, pilgrimage, Portugal, anthropology, religion, catholicism

\section{Contando Santo António em Lisboa. Atores, performances e efeitos do discurso hagiográfico contemporâneo}

Resumo : Este artigo examina as modalidades contemporâneas da narração da vida de Santo António em Lisboa (Portugal), no santuário onde se acredita 0 santo ter nascido. Através de uma etnografia das visitas conduzidas por vários atores (Franciscanos, guias turísticos, pessoal dos serviços culturais do municipio, diretor do museu Santo António), tentamos compreender como é que o santo universal de Pádua se torna num santo português. Se as sequências do nascimento e da formação religiosa do santo em Portugal formam parte essencial do processo de localização, as narrativas sobre as devoçôes populares em contexto português são igualmente importantes na construção desta imagem local. Assim, quer os temas hagiográficos quer as particula- 
ridades devocionais parecem apagar a figura eclesiástica, intelectual e universal do Santo António Doutor da Igreja valorizando, pelo contrário, a figura do intercessor local, popular e familiar do povo português. Por outro lado, a diversidade dos atores envolvidos na transmissão da vida do santo permite questionar a sobreposição dos motivos religiosos, turísticos e patrimoniais que cada um tenciona destacar através das diferentes figuras do santo, que aparece ora como modelo de vida cristã, ora como paradigma da religião popular, ora como simbolo da identidade nacional. O texto mostra a profunda imbricação do turismo, do património e da prática religiosa no contexto católico, que reclama uma abordagem mais aberta do fato religioso, deste modo colocando em evidência a diversidade das motivações associadas à religião no mundo atual.

Palavras-chave: santo, narrativas, turismo, peregrinação, Portugal, antropologia, religião, catolicismo

\section{Note sur l'auteur}

Cyril Isnart est anthropologue, chargé de recherche au CNRS (UMR 7307 IDEMEC CNRS AMU). Après un travail d'anthropologie religieuse dans les Alpes françaises et italiennes, il s'interroge aujourd'hui sur les conditions et les acteurs de la mise en patrimoine de la religion et de la musique en Europe du Sud. 